Review Article

\title{
Efficacy and Safety of Acupoint Catgut Embedding for Diarrhea-Predominant Irritable Bowel Syndrome and Constipation-Predominant Irritable Bowel Syndrome: A Systematic Review and Meta-Analysis
}

\author{
Jing Wu, ${ }^{1}$ Qinwei Fu $\mathbb{D},{ }^{2}$ Shasha Yang $\mathbb{D}^{3},{ }^{3}$ Hui Wang, ${ }^{1}$ and Yaofeng Li $^{1}$ \\ ${ }^{1}$ Guizhou University of Traditional Chinese Medicine, Guiyang 550025, China \\ ${ }^{2}$ Hospital of Chengdu University of Traditional Chinese Medicine, Chengdu University of Traditional Chinese Medicine, \\ Chengdu 610072, China \\ ${ }^{3}$ The First Affiliated Hospital of Guizhou University of Traditional Chinese Medicine, Guiyang 550002, China
}

Correspondence should be addressed to Shasha Yang; yangyuansha88@163.com

Received 29 May 2020; Revised 27 October 2020; Accepted 15 November 2020; Published 28 November 2020

Academic Editor: Tarique Hussain

Copyright $\odot 2020$ Jing Wu et al. This is an open access article distributed under the Creative Commons Attribution License, which permits unrestricted use, distribution, and reproduction in any medium, provided the original work is properly cited.

\begin{abstract}
In this study, we aim to evaluate the efficacy and safety of acupoint catgut embedding for the treatment of diarrhea-predominant irritable bowel syndrome and constipation-predominant irritable bowel syndrome. We searched seven online databases to collect studies published up to Feb $29^{\text {th }}, 2020$. Study quality of each included article was evaluated by the Cochrane Collaboration Risk of Bias Tool. Systematic reviews and meta-analyses were conducted based on the Cochrane systematic review method by using RevMan 5.3 software. Among the included trials, acupoint catgut embedding alone or plus oral western medicine or plus other acupoint-based therapies, or plus oral traditional Chinese medicine were the main therapies in the experimental groups. Interventions in control groups mainly include oral western medicine alone, other acupoint-based therapies alone, or other acupoint-based therapies alone. Primary outcomes in this study include recovery rate, accumulative marked effective rate, accumulative effective rate, and recurrence rate. Finally, 30 trials involving 1889 participants were included. The results of systematic reviews and meta-analyses show that acupoint catgut embedding alone or plus oral western medicine or plus other acupoint-based therapy or plus oral traditional Chinese medicine was significantly better compared with using oral western medicine alone in terms of efficacy for IBS-C and IBS-D. In addition, acupoint catgut embedding alone or plus oral western medicine or plus other acupoint-based therapy or plus oral traditional Chinese medicine could improve the effective rate and decrease the recurrence rate for IBS-D compared with using oral western medicine, other acupoint-based therapies, or oral traditional Chinese medicine alone. Adverse events of acupoint catgut embedding include local induration, redness, swelling, and itchiness, but they were all mild and disappeared swiftly with ordinary local interventions. There is an urgent need for RCTs of high quality and large sample size and with longer treatment duration and follow-up periods of acupoint catgut embedding for IBS.
\end{abstract}

\section{Introduction}

Considered the most common functional gastrointestinal disorder worldwide, irritable bowel syndrome (IBS) is characterized by chronic and recurrent abdominal pain and (or) altered bowel habits, which could not be explained by any anatomical or structural abnormality $[1,2]$.

According to Rome IV criteria, IBS is divided into four subtypes in view of symptoms, including diarrhea- predominant IBS (IBS-D), constipation-predominant IBS (IBS-C), IBS with mixed symptoms of diarrhea and constipation (IBS-M), and untyped IBS (IBS-U) [1]. Gastrointestinal tract motility, gut microbiotic imbalance, and visceral hypersensitivity contribute to IBS mainly, while more researches on its mechanisms are still needed urgently [3].

As of 2014, more than 23\% of the world population was suffering from IBS with decreased work productivity and life quality [4]. The international overall medical costs 
concerning IBS were more than 200 billion dollars in 2008, with potential indirect burden [5]. Conventional drugs, such as fiber supplementation, antispasmodics, probiotics, antidepressants, and psychological treatments, are used to alleviate the symptoms, but the effects are limited and followed by various adverse events [6-8].

Evidence from some RCTs favored several traditional Chinese medicine (TCM) therapies for IBS, such as TCM herbal decoctions, acupuncture, and moxibustion, with a considerable efficacy, lower recurrence rate, and fewer adverse events [9-11]. As a kind of TCM external therapy, acupoint catgut embedding (ACE) was a combination of TCM acupuncture theory (based on meridians and acupoints) and modern technologies (catgut embedding with special syringe), which is being applied widely especially in China. In addition, it is easier to operate and has more durable stimulation compared with acupuncture [12]. However, the use of ACE in the treatment of IBS beyond China is not popular, and the clinical efficacy of ACE alone or with other therapies is not certain.

Several reviews concerning TCM internal (TCM herbal medicine decoctions) and external (acupuncture and moxibustion) therapies for IBS have been published [13-20], while no study on the efficacy and safety of ACE for IBS has been conducted. The aim of our study is to identify the clinical efficacy and safety of ACE for the treatment of IBS-C and IBS$\mathrm{D}$ and to compare the efficacy and adverse effects of applying OWM, OTCM, or other ABT alone by several comparisons.

\section{Material and Methods}

2.1. Protocol and Registration. This systematic review was registered in PROSPERO, an international prospective register of systematic reviews, with the registration number CRD42020163031 (available from https://www.crd.york.ac. uk/prospero/display_record.php?RecordID=163031).

2.2. Search Strategy. We searched seven electronic databases, including Embase, PubMed, Cochrane Library, the China National Knowledge Infrastructure (CNKI), Technology Periodical Database (VIP), Wanfang Data Information Site, and SinoMed $(\mathrm{CBM})$ up to Feb $29^{\text {th }}, 2020$. The search strategy and inclusion criteria were decided according to the guidance of the PRISMA agreement. We used the following two groups of search terms in English: (1) "irritable bowel syndrome," "IBS" connected with "OR"; (2) "acupoint catgut embedding," "catgut embedding therapy," "catgut implantation at acupoint," "point embedding therapy" connected with "OR." The previously mentioned search terms of (1) and (2) were connected with term "AND." All searches were limited to trials of RCT in humans and were conducted in electronic databases by two authors independently. We also searched with related search terms in Chinese and searched the references of the original and review articles manually for possible related trials and also tried to get grey literatures identified through other sources.

2.3. Inclusion Criteria. In this systematic review, we searched and included trials according to the following criteria:
(1) Trials with participants that were diagnosed with IBS-D or IBS-C according to certain guidelines were included.

(2) Prospective randomized controlled trials (RCTs) were included.

(3) Trials in which acupoint catgut embedding alone or plus other therapy(ies) were applied in experimental groups. The patients in control groups received conventional therapy(ies), other TCM therapies, or placebo regimens. Trials for IBS-D or IBS-C combined with other disease(s) or for IBS but without classification of certain type (IBS-D or IBS-C) were excluded.

(4) Primary outcomes included recovery rate, accumulative marked effective rate, accumulative effective rate, and recurrence rate.

(5) Trials in Chinese or English were included.

2.4. Study Selection and Data Extraction. According to the aforementioned design, two reviewers (Jing Wu and Qinwei $\mathrm{Fu}$ ) searched the online databases listed previously and recorded the titles and abstracts of all the articles. Two evaluators (Hui Wang and Yaofeng Li) assessed the eligibility of these articles and made decisions on every research (inclusion or exclusion) independently. If they did not reach the same decision, the concerned articles were discussed with a fourth reviewer (Shasha Yang). Two reviewers (Jing $\mathrm{Wu}$ and Qinwei $\mathrm{Fu}$ ) extracted the data independently from each study. Differences in the extracted data were solved after discussion with a fourth reviewer (Shasha Yang).

2.5. Quality Assessment. Quality assessment of all the trials included in this review was independently evaluated by three reviewers (Jing Wu, Hui Wang, and Qinwei $\mathrm{Fu}$ ) using the Cochrane Collaboration Risk of Bias Tool by RevMan 5.3 software. Any disagreement was resolved by discussions with a fourth reviewer (Shasha Yang).

2.6. Statistical Analysis. This systematic review and metaanalysis was performed with the RevMan 5.3 software. Recovery rate, accumulative marked effective rate, accumulative effective rate, and recurrence rate were considered as dichotomous data, and some findings such as abdominal pain score, abdominal distention score, anorectal resting pressure, rectal maximum tolerance capacity, defecation frequency score, and mucinous stool score were considered as continuous data. Risk ratio (RR) and mean difference (MD) with 95\% confidence intervals (CIs) were given separately, which was an estimate of the pooled effect sizes, and $P$ values of less than 0.05 were considered statistically significant.

For the assessment of heterogeneity, we evaluated trials using both the $I^{2}$ statistic and Chi-square test $(P<0.1)$, which indicates the proportion of variability across trials not explained by sampling variation alone, and the $P$ value of the $V^{2}$ test of heterogeneity. 
$P<0.1$ or $I^{2}>50 \%$ indicated significant heterogeneity. If significant heterogeneity was not observed, a fixed-effects model was used to make estimates; otherwise, a randomeffects model was applied to statistical analysis.

Exploration of publication bias was planned if more than ten trials were included. Due to the number of included trials and methodological quality, not all planned analyses could be available.

\section{Results}

3.1. Study Inclusion. Initially, 293 records were searched from seven databases with no grey literature reference. After the removal of duplicates, the records were decreased to 87 . Based on the titles and abstracts of records, we excluded 31 papers with reasons such as observational studies, case reports, uncontrolled studies, animal experiments, reviews, and studies with no randomization-control design and not related to acupoint catgut embedding for IBS-D or IBS-C. The remaining 56 articles were downloaded for further selection, and 30 articles were excluded with reasons (Figure S1). Eventually, 30 trials form 26 studies (one fourarm study was recombined to five trials for comparison) were included [21-46]. The flow diagram of the study selection process is shown in Figure S1.

3.2. Study Characteristics. All 30 included RCTs were conducted in China and published in Chinese, with the range of publish years from 2008 to 2019 [21-46]. In total, 699 participants (11 trials) aging from 16 to 75 with IBS-C from three months to 12 years and 1190 participants (19 trials) aging from 18 to 69 with IBS-D from 15 days to 22 years were involved. Details of baseline characteristics were not reported in several trials, but no significant difference between groups $(P<0.05)$ among the characteristics was mentioned in all of them. As for the interventions of experimental groups, ACE alone, or combined with OTCM (TCM decoction/powder/granule) or with other ABT (e.g., auricular therapy, acupressure therapy for IBS-C, and fire needle therapy, moxibustion, and acupoint application for IBS-D) or with OWM (same as the medicine used in the control groups mostly), was applied in both IBS-C and IBS-D. In addition, ACE plus Tai Chi Chuan or diet therapy was applied in two trials (IBS-C) separately [36, 43]. Though some of the specific prescriptions of the TCM decoction/ powder/granule applied were different among the included trials, their principles and theories showed similarity according to the theory of TCM. In addition, OWM alone was applied in control groups of 10 trials for IBS-C [21, 36-42, 44-46], with OWM plus sham ACE in one trial [43]. As for the control groups for IBS-D, OWM alone was applied in 11 trials $[22,24,25,27,29,31-35]$, TCM decoction/powder in five trials $[23,26,28,33]$, other ABT (acupuncture and acupoint application) in two trials $[21,30]$, and ACE plus Buzhong Yiqi decoction (TCM) in one trial [33]. Detailed characteristics of the included trials are listed in Tables S1 and S2.
3.3. Assessment of Quality and Bias. According to the results of Cochrane Collaboration Risk of Bias Tool [47], eight trials described the method of randomization clearly and appropriately with no trial in high risk of bias $[22,26,27,31,35,37,38,43]$. The method of allocation concealment was described clearly in four trials $[26,35,37,38]$ but was unclear in the others. No trial reported blinding method in addition to one study with sham ACE applied in the control groups [43]. Specially assigned procedures with blinding for outcome assessment were applied in six trials [24, 26, 35, 38, 42, 46], and two trials reported protocol or registration ahead of experiment $[38,43]$. The details are shown in Figures 1 and 2.

\subsection{Efficacy of ACE in AR Patients}

3.4.1. ACE Alone versus OWM Alone (for IBS-C and IBS-D). Studies included favored ACE alone for both IBS-C [37, 44] and IBS-D [24, 25, 33, 34] on higher recovery rate, accumulative marked effective rate, and accumulative effective rate significantly $(P<0.05)$, with none to mild heterogeneity $\left(I^{2}: 0 \%-8 \%\right)$ (Tables 1 and 2; Figures S2 and S4). More and significant $(P<0.05)$ reductions on abdominal pain score $(\mathrm{MD}=-0.53 /-0.75)$ were reported in ACE alone groups for both IBS-C [37, 44] and IBS-D [25, 33], but with considerable heterogeneity $\left(I^{2}: 58 \%-76 \%\right)$ (Figures S3 and 5).

In addition, evidence shows that ACE alone could better relieve abdominal distention for IBS-C patients $(\mathrm{MD}=-0.26, P<0.05)$ with no heterogeneity $[37,44]$ and decrease recurrence rate after three months $(\mathrm{MD}=0.49, P$ for $\mathrm{MD}>0.05, \quad I^{2}=60 \%$ ) and defecation frequency $\left(\mathrm{MD}=-0.93, P\right.$ for $\left.\mathrm{MD}>0.05, I^{2}=99 \%\right)$ for patients with IBS-D compared with the control groups $[25,33]$ (Figures S2 and S5). However, results show that ACE alone could not improve mucinous stool better compared with applying $\mathrm{WM}$ alone $(\mathrm{MD}=0.13, P$ for $\mathrm{MD}>0.05)$ for patients with IBS-D with mild heterogeneity $\left(I^{2}=15 \%\right) \quad[25,33]$ (Figure S5).

3.4.2. ACE plus Other ABT versus OWM Alone (for IBS-C and IBS-D). With regard to ACE plus other ABT groups compared with OWM alone groups, evidence favored higher accumulative marked effective rate (IBS-C: $\mathrm{RR}=1.44 ; P$ for RR $>0.05 ; 95 \%$ CI: $0.82-2.51 ; I^{2}=$ not applicable; IBS-D: $\mathrm{RR}=1.8 ; P$ for $\left.\mathrm{RR}<0.01 ; 95 \% \mathrm{CI}: 1.23-2.62 ; I^{2}=0 \%\right)$ and accumulative effective rate (IBS-C: $\mathrm{RR}=1.27 ; P$ for $\mathrm{RR}<0.01 ; 95 \%$ CI: $1.08-1.49 ; I^{2}=0 \%$; IBS-D: RR $=1.33 ; P$ for $\left.\mathrm{RR}<0.01 ; 95 \% \mathrm{CI}: 1.09-1.62 ; I^{2}=35 \%\right)$ for patients with IBSC $[38,40,45]$ or IBS-D $[29,32]$, respectively. Results also show that ACE plus other ABT could provide significantly higher recovery rate for patients with IBS-C $(\mathrm{RR}=2.73 ; P$ for $\mathrm{RR}=0.01 ; 95 \% \mathrm{CI}: 1.22-6.09 ; I^{2}=0 \%$ ) [38, 40, 45] (Tables 1 and 2; Figures S6 and S7).

3.4.3. ACE plus OTCM versus OWM Alone (for IBS-C and IBS-D). Findings of meta-analysis show that ACE plus OTCM could relieve constipation symptoms better for 


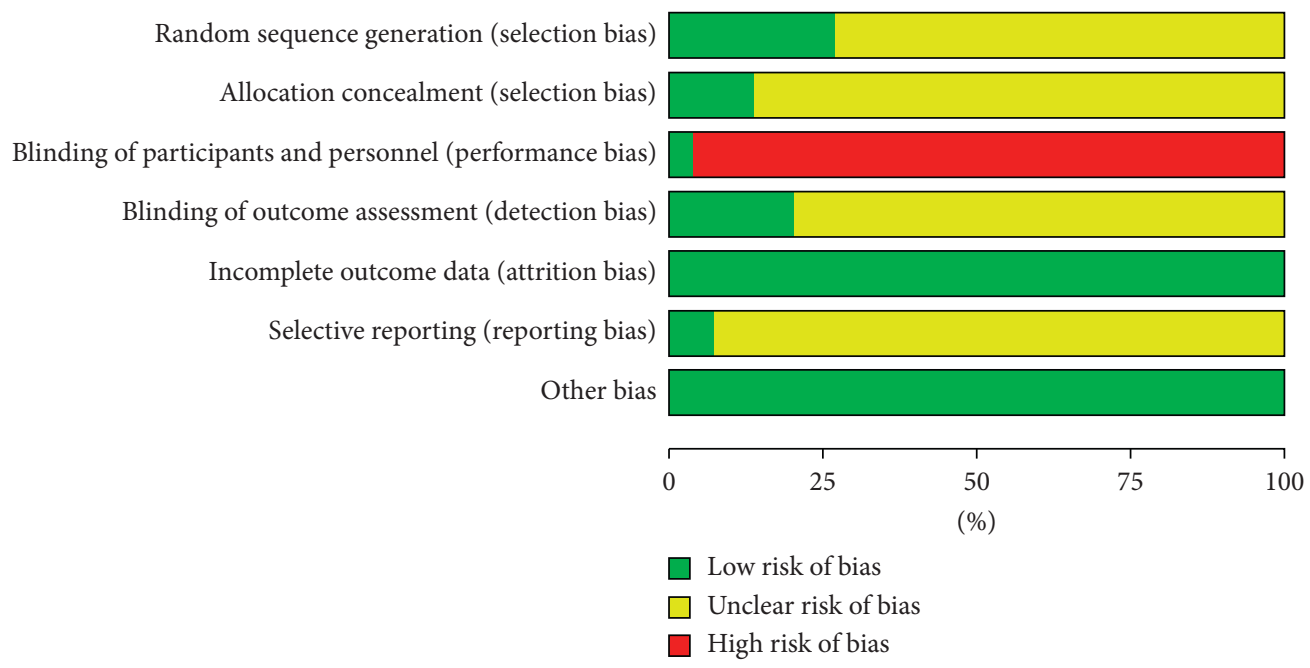

FIGURE 1: Risk of bias graph.

IBS-C patients compared with OWM alone, including lower anorectal resting pressure $(\mathrm{MD}=-2.81 ; P$ for $\mathrm{MD}=0.03$; 95\% CI: -5.32 to $\left.-0.3 ; I^{2}=0 \%\right)$ and rectal maximum tolerance capacity $(\mathrm{MD}=-19.3 ; P$ for $\mathrm{MD}<0.01 ; 95 \% \mathrm{CI}:-32.56$ to $-6.04 ; I^{2}=77 \%$ ) $[41,46]$ (Table 1; Figure S8).

In addition, by comparisons of ACE plus OTCM versus OWM alone, the pooled results favored the experimental groups on recovery rate $(\mathrm{RR}=3.36$; $P$ for $\mathrm{RR}=0.29$; $95 \% \mathrm{CI}$ : $\left.0.35-31.93 ; I^{2}=77 \%\right)$, accumulative marked improvement rate $\left(\mathrm{RR}=1.87 ; P\right.$ for $\left.\mathrm{RR}<0.01 ; 95 \% \mathrm{CI}: 1.24-2.81 ; I^{2}=47 \%\right)$, and accumulative effective rate $(\mathrm{RR}=1.31 ; P$ for $\mathrm{RR}<0.01$; 95\% CI: $\left.1.15-1.5 ; I^{2}=0 \%\right)$ in three trials for IBS-D [22, 27, 33] (Table 2; Figure S9).

3.4.4. ACE plus OTCM versus OTCM Alone (for IBS-D Only). Results of systematic review demonstrate that ACE plus OTCM could provide better improvements than OTCM alone without heterogeneity for patients with IBS-D (Table 2$)$, including recovery rate $(\mathrm{RR}=1.82 ; P$ for $\mathrm{RR}=0.01$; 95\% CI: 1.14-2.92) [23, 33], accumulative marked improvement rate $(\mathrm{RR}=1.31 ; P$ for $\mathrm{RR}=0.07 ; 95 \% \mathrm{CI}$ : $0.98-1.74)[28,33]$ accumulative effective rate $(\mathrm{RR}=1.11 ; P$ for $\mathrm{RR}=0.07$; 95\% CI: 0.99-1.24) [23, 28, 33], and recurrence rate $(6$ months $)(\mathrm{RR}=0.65 ; P$ for $\mathrm{RR}=0.2 ; 95 \% \mathrm{CI}$ : $0.33-1.26)[23,33]$ (Figure S10).

3.4.5. ACE plus Other ABT versus Other ABT Alone (for IBS$D$ Only). Compared with the other ABT groups, significant improvements were found in ACE plus other ABT groups (Table 2), including accumulative marked effective rate $\left(\mathrm{RR}=1.32 ; P\right.$ for $\left.\mathrm{RR}<0.01 ; 95 \% \mathrm{CI}: 1.12-1.56 ; I^{2}=0 \%\right)$ and accumulative effective rate in two trials $(\mathrm{RR}=1.23 ; P$ for $\mathrm{RR}<0.01 ; 95 \%$ CI: $1.11-1.35 ; I^{2}=0 \%$ ) [21, 30] (Figure S11).

3.4.6. ACE plus OWM versus OWM Alone (for IBS-D Only). Compared with the control groups, pooled results of two trials show favored ACE plus OWM concerning recovery rate $\left(\mathrm{RR}=2.72 ; P\right.$ for $\mathrm{RR}=0.12 ; 95 \% \mathrm{CI}: 0.76-9.69 ; I^{2}$ : not applicable), accumulative marked improvement rate $\left(\mathrm{RR}=5.09 ; P\right.$ for $\left.\mathrm{RR}=0.28 ; 95 \% \mathrm{CI}: 0.27-94.99 ; I^{2}=78 \%\right)$, and accumulative effective rate $(\mathrm{RR}=1.46 ; P$ for $\mathrm{RR}<0.01$; 95\% CI: $1.21-1.77 ; I^{2}=0 \%$ ) [31, 35] (Table 2; Figure S12).

3.5. Adverse Events Reported in Trials. Adverse events were only reported for the experimental groups of two trials $[26,35]$, with no adverse event reported in seven trials (neither in experimental groups nor in control groups). One trial reported local induration on the acupoints $(3$ cases, $5.08 \%$ ) after ACE, which was relieved after local normal acupuncture and hot compress [26]. Local redness, swelling, and itchiness for ACE were found in one trial, and the events disappeared after two weeks by local application of iodophor (tid) [35].

Adverse events were not reported in the other 21 trials.

\section{Discussion}

IBS is a chronic functional disorder with clinical severity, which varies from episodic mild pain up to severe daily symptoms [48]. There is no therapy with universal recognition at present, while an increasing number of patients and medical staff have turned to some complementary and alternative medicine therapies for treatment. Some evidence has proved the efficacy and safety of ACE for abdominal obesity, hypertension, diabetes and its chronic complications, postmenopausal osteoporosis, infertility, and allergic rhinitis [49-54]; however, there was no systematic review and meta-analysis of efficacy and safety for IBS. Our systematic review and meta-analysis firstly evaluated efficacy and safety of ACE for both IBS-C and IBS-D, respectively, for more general and comprehensive evidence.

ACE shares many similarities with TCM acupuncture, including theories of TCM, meridian especially, acupoint selection based on syndrome differentiation, and some operation requirements for acupoint-based therapies (e.g., De Q $i$ in TCM) [55]. In addition, there exist some differences in techniques and tools (catgut embedding by 


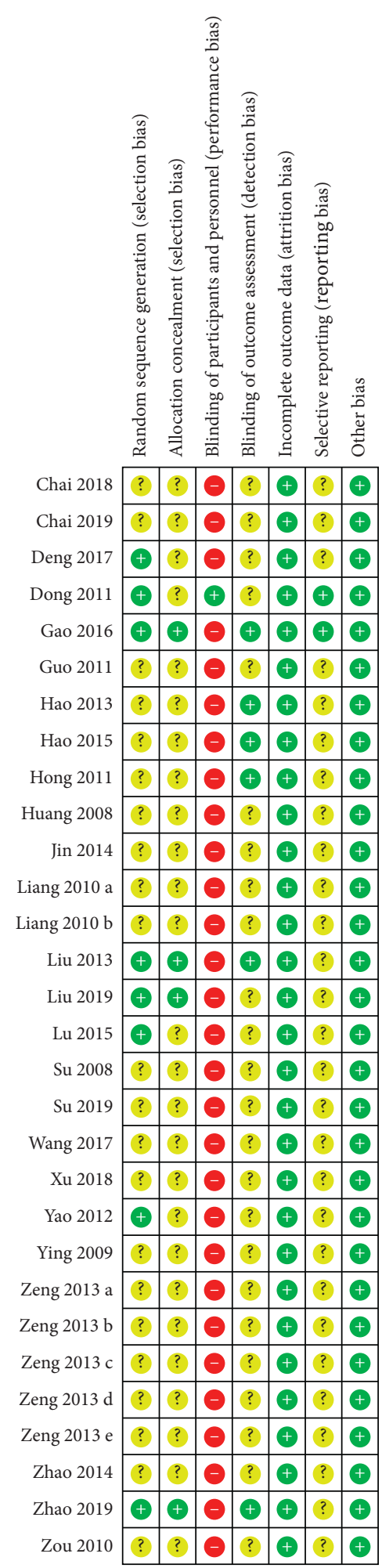

Figure 2: Risk of bias summary.

special syringe at the surface of the body, muscle layer usually, but not by acupuncture needle) and tendency of acupoint selection (acupoints where fat muscle exists, such as waist, back and abdomen which were more preferred, although ACE will be applied for some patients with intractable facial paralysis). Acupoints for acupuncture could be referred for acupoint selection process of ACE on the basis of this.

Results of our study indicate that, compared with OWM alone, pooled results of our study covering 20 trials with 1252 participants favored ACE alone (or plus other ABT or plus OTCM) concerning higher efficacy and lower recurrence rate (ACE alone vs. OWM alone for IBS-C) for IBS-C and IBS-D. In addition, ACE plus OTCM (or plus other ABT or plus OWM) exhibited favorable improvements compared with the control groups (OTCM, other ABT, or OWM alone correspondingly) for IBS-D.

In TCM theory, IBS-C and IBS-D are categorized as constipation disease and diarrhea disease, respectively, which is combined with abdominal pain disease sometimes. Syndromes of IBS fall into excess syndrome (liver depression and $Q i$ stagnation, stagnant heat of intestine, damp-heat in spleen and stomach), deficiency syndrome (spleen-kidney yang deficiency), and a combination of both (spleen deficiency with damp encumbrance, liver depression, and spleen deficiency) [56]. According to TCM theory, effects of ACE include balancing yin, yang, and $z a n g-f u$ organs, promoting meridian $Q i$, regulating $Q i$ and blood, tonifying for the deficiency and reducing for the excess, strengthening the antipathogenic $Q i$, and eliminating pathogens [55]. In addition, most of the OTCM included in our study have the effect of moving $Q i$ and removing food stagnation and are widely used in the treatment of IBS-C, or tonifying $Q i$, invigorating spleen and draining dampness and checking diarrhea for IBS-D according to TCM theories.

Evidence illustrates that some TCM therapies have "twoway adjusting effects" (for example, TCM herbal decoctions, acupuncture, and moxibustion) [57-60], and we briefly divided them into TCM internal therapies (e.g., TCM herbal decoctions and Chinese patent medicine) and TCM external therapies (e.g., acupuncture, moxibustion, acupoint application, and ACE). Modern researches show that ACE plays its role by recovering nerve function, regulating neural reflex, increasing human immunity, improving local circulation, inhibiting the release of inflammatory factors, reducing apoptosis, regulating cellular factor, and improving body metabolism [55]. For instance, evidence shows that ACE could be applied for diarrhea/constipation [24, 61].

Compared with other TCM therapies, ACE could provide patients with lower expense, shorter time of treatment, and longer stimulation sustention with fewer side effects such as local pain and hematoma [62]. As a result, ACE should be widely recognized and accepted for IBS in future. It should be also pointed out that several studies of high quality in recent years approved the efficacy and safety of some TCM external therapies, especially acupoint-based ones, in preventing and controlling some diseases, such as acupuncture for postprandial distress syndrome, acupuncture and acupressure for cancer pain, acupuncture for chronic stable angina, and acupressure combined with TCM footbath for diabetic peripheral neuropathy [63-66]. Such external therapies could reduce the intake of some medications, especially those that may be substantially addictive and with adverse events (e.g., opioid). 
TABLE 1: Summary of findings for IBS-C.

\begin{tabular}{|c|c|c|c|c|c|c|c|}
\hline Intervention & Outcome & $\begin{array}{c}\text { No. of } \\
\text { trials }\end{array}$ & Participants & $\begin{array}{l}\text { Effect size } \\
(\mathrm{RR} / \mathrm{MD})\end{array}$ & $95 \% \mathrm{CI}$ & $\begin{array}{l}P \text { value of effect } \\
\text { size }\end{array}$ & $\begin{array}{c}I^{2} \text { value } \\
(\%)\end{array}$ \\
\hline \multirow{5}{*}{ ACE vs. OWM } & Recovery rate & \multirow{5}{*}{2} & \multirow{5}{*}{198} & 5.50 & 1.99 to 15.17 & \multirow{5}{*}{$<0.001$} & \\
\hline & $\begin{array}{l}\text { Accumulative marked effective } \\
\text { rate }\end{array}$ & & & 3.46 & 2.03 to 5.90 & & 0 \\
\hline & Accumulative effective rate & & & 1.30 & 1.12 to 1.50 & & \\
\hline & Abdominal pain score & & & -0.53 & -0.55 to -0.51 & & 58 \\
\hline & Abdominal distention score & & & -0.26 & -0.29 to -0.23 & & 0 \\
\hline \multirow{3}{*}{$\mathrm{ACE}+\mathrm{OAT}$ vs. OWM } & \multirow{3}{*}{$\begin{array}{c}\text { Recovery rate } \\
\text { Accumulative marked effective } \\
\text { rate } \\
\text { Accumulative effective rate }\end{array}$} & 3 & 196 & 2.73 & 1.22 to 6.09 & 0.01 & 0 \\
\hline & & 1 & 68 & 1.44 & 0.82 to 2.51 & 0.2 & NA \\
\hline & & 3 & 196 & 1.27 & 1.08 to 1.49 & $<0.01$ & 0 \\
\hline \multirow[b]{2}{*}{$\begin{array}{l}\text { ACE + OTCM vs. } \\
\text { OWM }\end{array}$} & \multirow{2}{*}{$\begin{array}{l}\text { Anorectal resting pressure } \\
\text { Rectal maximum tolerance } \\
\text { capacity }\end{array}$} & \multirow[b]{2}{*}{2} & \multirow[b]{2}{*}{110} & -2.81 & -5.32 to -0.3 & 0.03 & 0 \\
\hline & & & & -19.3 & $\begin{array}{c}-32.56 \text { to } \\
-6.04\end{array}$ & $<0.01$ & 77 \\
\hline
\end{tabular}

ACE: acupoint catgut embedding; OWM: oral western medicine; OAT: other acupoint therapies; OTCM: oral traditional Chinese medicine; NA: not applicable.

TABLE 2: Summary of findings for IBS-D.

\begin{tabular}{|c|c|c|c|c|c|c|c|}
\hline Intervention & Outcome & $\begin{array}{c}\text { No. of } \\
\text { trials }\end{array}$ & Participants & $\begin{array}{l}\text { Effect size } \\
(\mathrm{RR} / \mathrm{MD})\end{array}$ & $95 \% \mathrm{CI}$ & $\begin{array}{l}P \text { value of } \\
\text { effect size }\end{array}$ & $I^{2}$ value $(\%)$ \\
\hline \multirow{7}{*}{ ACE vs. OWM } & Recovery rate & 3 & 181 & 2.16 & 1.33 to 3.53 & $<0.01$ & 0 \\
\hline & $\begin{array}{c}\text { Accumulative marked effective } \\
\text { rate }\end{array}$ & \multirow[t]{2}{*}{4} & \multirow[t]{2}{*}{241} & 1.44 & 1.14 to 1.83 & $<0.01$ & 8 \\
\hline & Accumulative effective rate & & & 1.27 & 1.12 to 1.44 & $<0.01$ & 0 \\
\hline & Recurrence rate (3 months) & 2 & 101 & 0.49 & 0.07 to 3.24 & 0.46 & 60 \\
\hline & Abdominal pain score & \multirow{3}{*}{2} & \multirow{3}{*}{121} & -0.75 & -1.41 to -0.1 & 0.02 & 76 \\
\hline & Defecation frequency score & & & -0.93 & -3.52 to 1.65 & 0.48 & 99 \\
\hline & Mucinous stool score & & & 0.13 & -0.08 to 0.33 & 0.23 & 15 \\
\hline \multirow[t]{2}{*}{$\mathrm{ACE}+\mathrm{OAT}$ vs. OWM } & $\begin{array}{l}\text { Accumulative marked effective } \\
\text { rate }\end{array}$ & \multirow[t]{2}{*}{2} & \multirow[t]{2}{*}{131} & 1.8 & 1.23 to 2.62 & $<0.01$ & 0 \\
\hline & Accumulative effective rate & & & 1.33 & 1.09 to 1.62 & $<0.01$ & 35 \\
\hline \multirow{3}{*}{ ACE + OTCM vs. OWM } & Recovery rate & \multirow{3}{*}{3} & \multirow{3}{*}{191} & 3.36 & 0.35 to 31.93 & 0.29 & 77 \\
\hline & $\begin{array}{l}\text { Accumulative marked effective } \\
\text { rate }\end{array}$ & & & 1.87 & 1.24 to 2.81 & $<0.01$ & 47 \\
\hline & Accumulative effective rate & & & 1.31 & 1.15 to 1.5 & $<0.01$ & 0 \\
\hline \multirow{4}{*}{$\begin{array}{l}\text { ACE + OTCM vs. } \\
\text { OTCM }\end{array}$} & \multirow{4}{*}{$\begin{array}{c}\text { Recovery rate } \\
\text { Accumulative marked effective } \\
\text { rate } \\
\text { Accumulative effective rate } \\
\text { Recurrence rate ( } 6 \text { months) }\end{array}$} & 2 & 120 & 1.82 & 1.14 to 2.92 & 0.01 & 0 \\
\hline & & 2 & 126 & 1.31 & 0.98 to 1.74 & 0.07 & 0 \\
\hline & & 3 & 206 & 1.11 & 0.99 to 1.24 & 0.07 & 0 \\
\hline & & 2 & 120 & 0.65 & 0.33 to 1.26 & 0.2 & 0 \\
\hline \multirow[t]{2}{*}{$\mathrm{ACE}+\mathrm{OAT}$ vs. OAT } & $\begin{array}{l}\text { Accumulative marked effective } \\
\text { rate }\end{array}$ & \multirow[t]{2}{*}{2} & \multirow[t]{2}{*}{248} & 1.32 & 1.12 to 1.56 & $<0.01$ & 0 \\
\hline & Accumulative effective rate & & & 1.23 & 1.11 to 1.35 & $<0.01$ & 0 \\
\hline \multirow{3}{*}{$\mathrm{ACE}+\mathrm{OWM}$ vs. OWM } & Recovery rate & \multirow{3}{*}{2} & \multirow{3}{*}{165} & 2.72 & 0.76 to 9.68 & 0.12 & Not applicable \\
\hline & $\begin{array}{l}\text { Accumulative marked effective } \\
\text { rate }\end{array}$ & & & 5.09 & 0.27 to 94.99 & 0.28 & 78 \\
\hline & Accumulative effective rate & & & 1.46 & 1.21 to 1.77 & $<0.01$ & 0 \\
\hline
\end{tabular}

ACE: acupoint catgut embedding; OWM: oral western medicine; OAT: other acupoint therapies; OTCM: oral traditional Chinese medicine; NA: not applicable.

In our study, only two of 30 trials reported adverse events related to ACE, and no adverse event was reported on other interventions. The adverse events of ACE were local induration, redness, swelling, and itchiness, which disappeared swiftly after local normal acupuncture and hot compress or local application of iodophor. Results of Wang and colleagues demonstrated that adverse events were found in 70 of 331 patients (21.1\%) who seek ACE, including discomfort, body temperature rising after treatment, local hematoma, subcutaneous hemorrhage, swelling, induration, pain, pruritus, redness, and fever [67]. Fainting during the treatment of ACE (1 case) was reported in one trial [68].

As for study quality and risk of bias, all the 30 trials are RCTs, but only one of them implied placebo control. 
Randomization method was clear and appropriate in eight trials, while it was of unclear risk of bias for the other 22 trials. Allocation concealment and blinding method were of unclear risk of bias in most trials. No study reported dropout, and a protocol or registration ahead of experiment was reported in only two trials. As a result, more double-blind, prospective, randomized, placebo-controlled trials of ACE as a therapy for IBS with different subtypes are urgently needed.

\section{Limitations}

There are several limitations in our systematic review and meta-analysis. First, most of the trials included were of moderate-to-high risk of bias, with reasons, such as, without mentioning details, random sequence generation method, allocation concealment, and blinding of participants, personnel, and outcome assessment. This is the main reason for low quality of the included trials. Second, interventions and follow-up periods were short among most of the trials, while longer treatment duration and follow-up periods for IBS, a chronic and recurrent disorder, are essential and required. Finally, assessment of publication bias was not applicable in our study, for no more than 10 trials meeting the criteria were included in each comparison.

Generally, more RCTs of high quality and large sample size and with longer treatment duration and follow-up periods are needed to further improve and update our evidence.

\section{Conclusion}

This systematic review and meta-analysis demonstrated that applying ACE alone or plus OWM or plus other ABT or plus OTCM may be more effective for the treatment of IBS-C and IBS-D than OWM alone. ACE plus OTCM or plus other ABT or plus OWM exhibited favorable improvements compared with the control groups (OTCM, other ABT, or OWM alone correspondingly) for IBS-D. Adverse events of ACE were local induration, redness, swelling, and itchiness, but they were all mild and disappeared swiftly with ordinary local intervention. There is an urgent need for RCTs of high quality and large sample size and with longer treatment duration and follow-up periods of ACE for IBS.

\section{Abbreviations}

IBS-D: Diarrhea-predominant irritable bowel syndrome

IBS-C: Constipation-predominant irritable bowel syndrome

ACE: Acupoint catgut embedding

OWM: Oral western medicine

ABT: Acupoint-based therapy

OTCM: Oral traditional Chinese medicine

RR: $\quad$ Risk ratio

MD: $\quad$ Mean difference

CI: Confidence interval.

\section{Data Availability}

The data are available upon request to the corresponding author.

\section{Disclosure}

Qinwei Fu is a co-first author.

\section{Conflicts of Interest}

There are no financial conflicts or other relationships for the authors to be declared.

\section{Authors' Contributions}

J Wu, QW Fu, SS Yang, and H Wang were involved in the conception and design. All the authors were involved in the analysis and interpretation of the data. Jing W, QW Fu, SS Yang, and YF Li were involved in the drafting of the paper or revising it critically for intellectual content. All authors agreed to be accountable for all aspects of the work.

\section{Acknowledgments}

This project was jointly supported by National Natural Science Foundation of China (no. 81960821); Doctoral Fund Project of the First Affiliated Hospital of Guizhou University of Traditional Chinese Medicine (GYZYYFY-BS-2019(01)); Youth Science and Technology Talent Growth Project of Guizhou Provincial Department of Education (Qian Jiao He KY [2017]170); and Research Project of Science and Technology of Traditional Chinese Medicine and National Medicine of Guizhou Administration of Traditional Chinese Medicine (QZYY-2018-026).

\section{Supplementary Materials}

Detailed characteristics of included trials (IBS-C and IBS-D), the flow diagram of the study selection process, and forest plots of the comparisons in our study are listed in supplementary materials document. (Supplementary Materials)

\section{References}

[1] G. F. Longstreth, W. G. Thompson, W. D. Chey, L. A. Houghton, F. Mearin, and R. C. Spiller, "Functional bowel disorders," Gastroenterology, vol. 130, no. 5, pp. 1480-1491, 2006.

[2] L. J. Brandt, W. D. Chey, A. E. Foxx-Orenstein et al., "An evidence-based systematic review on the management of irritable bowel syndrome," American Journal of Gastroenterology, vol. 104, no. Suppl 1, p. S8, 2009.

[3] A. C. Ford and N. J. Talley, "Advances in pathophysiology, diagnosis and treatment," Nature Reviews Gastroenterology \& Hepatology, vol. 8, no. 2, pp. 76-78, 2011.

[4] L. Saha, "Irritable bowel syndrome: pathogenesis, diagnosis, treatment, and evidence-based medicine," World Journal of Gastroenterology, vol. 20, no. 22, pp. 6759-6773, 2014.

[5] L. V. McFarland, "State-of-the-art of irritable bowel syndrome and inflammatory bowel disease research in 2008," World 
Journal of Gastroenterology, vol. 14, no. 17, pp. 2625-2629, 2008.

[6] A. Annaházi, R. Róka, A. Rosztóczy, and T. Wittmann, "Role of antispasmodics in the treatment of irritable bowel syndrome," World Journal of Gastroenterology, vol. 20, no. 20, pp. 6031-6043, 2014.

[7] C. Xie, Y. Tang, Y. Wang et al., "Efficacy and safety of antidepressants for the treatment of irritable bowel syndrome: a meta-analysis," PLoS ONE, vol. 10, no. 8, Article ID e0127815, 2015.

[8] I. L. Zijdenbos, N. J. de Wit, G. J. van der Heijden, G. Rubin, and A. O. Quartero, "Psychological treatments for the management of irritable bowel syndrome," The Cochrane Database of Systematic Reviews, vol. 1, Article ID CD006442, 2009.

[9] X.-d. Tang, S.-s. Zhang, X.-h. Hou et al., "Post-marketing Reevaluation of tongxiening granules (痛泻宁颗粒) in treatment of diarrhea-predominant irritable bowel syndrome: a multi-center, randomized, double-blind, double-dummy and positive control trial," Chinese Journal of Integrative Medicine, vol. 25, no. 12, p. 887, 2019.

[10] J.-m. Zhao, J.-h. Lu, X.-j. Yin et al., "Comparison of electroacupuncture and mild-warm moxibustion on brain-gut function in patients with constipation-predominant irritable bowel syndrome: a randomized controlled trial," Chinese Journal of Integrative Medicine, vol. 24, no. 5, p. 328, 2018.

[11] Q. Mao, L. Shi, Z.-G. Wang et al., "Chemical profiles and pharmacological activities of Chang-Kang-Fang, a multi-herb Chinese medicinal formula, for treating irritable bowel syndrome," Journal of Ethnopharmacology, vol. 201, pp. 123-135, 2017.

[12] L.-j. Deng, Z.-j. Lun, X.-w. Ma, and J.-l. Zhou, "Clinical observation on regulating the three energizer by acupoint catgut embedding combined with abdominal acupuncture in treating abdominal obesity: a randomized controlled trial," World Journal of Acupuncture-Moxibustion, vol. 24, no. 4, pp. 29-34, 2014.

[13] J.-J. Zhu, S. Liu, X.-L. Su et al., "Efficacy of Chinese herbal medicine for diarrhea-predominant irritable bowel syndrome: a meta-analysis of randomized, double-blind, placebo-controlled trials," Evidence-Based Complementary and Alternative Medicine, vol. 2016, Article ID 4071260, 15 pages, 2016.

[14] Y. Zhou, S. Han, and Y. He, "Clinical effects and safety of tongxieyaofang on diarrhea predominant irritable bowel syndrome: a meta-analysis of randomized trails," EvidenceBased Complementary and Alternative Medicine, vol. 2019, Article ID 4893876, 11 pages, 2019.

[15] H. Zheng, R. Chen, X. Zhao et al., "Comparison between the effects of acupuncture relative to other controls on irritable bowel syndrome: a meta-analysis," Pain Research and Management, vol. 2019, Article ID 2871505, 13 pages, 2019.

[16] J. Shi, Y. Tong, J.-G. Shen, and H.-X. Li, "Effectiveness and safety of herbal medicines in the treatment of irritable bowel syndrome: a systematic review," World Journal of Gastroenterology, vol. 14, no. 3, p. 454, 2008.

[17] B. Tang, J. Zhang, Z. Yang et al., "Moxibustion for diarrheapredominant irritable bowel syndrome: a systematic review and meta-analysis of randomized controlled trials," EvidenceBased Complementary and Alternative Medicine, vol. 2016, Article ID 5105108, 10 pages, 2016.

[18] D.-y. Li, Y.-k. Dai, Y.-z. Zhang et al., "Systematic review and meta-analysis of traditional Chinese medicine in the treatment of constipation-predominant irritable bowel syndrome," PLoS One, vol. 12, no. 12, Article ID e0189491, 2017.

[19] J. Yan, Z.-w. Miao, J. Lu et al., "Acupuncture plus Chinese herbal medicine for irritable bowel syndrome with diarrhea: a systematic review and meta-analysis," Evidence-Based Complementary and Alternative Medicine, vol. 2019, Article ID 7680963, 16 pages, 2019.

[20] L. Dai, L. L. Zhong, and G. Ji, "Irritable bowel syndrome and functional constipation management with integrative medicine: a systematic review," World Journal of Clinical Cases, vol. 7, no. 21, pp. 3486-3504, 2019.

[21] Z. Chai, C. Zang, R. Wei, and L. He, "To explore the clinical effect of fire needle acupuncture combined with acupoint catgut embedding on irritable bowel syndrome (diarrhea type) of deficiency of spleen and kidney yang," Journal of Colorectal \& Anal Surgery, vol. 24, no. S2, pp. 59-60, 2018, in Chinese.

[22] X. Deng and Z. Peng, "Treatment of 30 cases of diarrhea type irritable bowel syndrome with compound Zhixie powder and Catgut Embedding at acupoints," Hunan Journal of Traditional Chinese Medicine, vol. 33, no. 1, pp. 53-54, 2017, in Chinese.

[23] J. Guo, "40 cases of diarrhea type irritable bowel syndrome treated by Tongxieyaofang Decoction and Catgut Embedding at acupoints," Zhejiang Journal of Traditional Chinese Medicine, vol. 4, p. 256, 2011, in Chinese.

[24] Z. Hong, Z. Wang, and X. Chen, "Therapeutic effect of acupoint catgut embedding on irritable bowel syndrome of diarrhea type," Chinese Acupuncture \& Moxibustion, vol. 4, pp. 311-313, 2011, in Chinese.

[25] S. Huang, S. Chen, and S. Lin, "Clinical observation on diarrhea irritable bowel syndrome by the treatment of burying line nearby spinal column echo area," Journal of Liaoning University of Traditional Chinese Medicine, vol. 7, pp. 86-87, 2008, in Chinese.

[26] Q. Liu, "Clinical research on irritable bowel syndrome of the type of diarrhea (liver Qi over-acting spleen) treated by acupoint catgut embedding therapy," Master's Thesis, Guangzhou University of Chinese Medicine, Guangzhou, China, 2013, in Chinese.

[27] C. Lu, "Treating IBS with catgut embedding plus free-fried TCM medicine granule," Clinical Journal of Chinese Medicine, vol. 7, no. 2, pp. 27-28, 2015, in Chinese.

[28] Q. Su, X. Qian, and X. Qian, "86 cases of diarrhea type irritable bowel syndrome treated with Anchang Yin Decoction and acupoint minimally invasive catgut embedding," Journal of Emergency in Traditional Chinese Medicine, vol. 11, pp. 1614-1615, 2008, in Chinese.

[29] Q. Su, Y. Wang, Y. Wen, Y. Guo, and X. Chen, "Clinical study on du-moxibustion combined with point embedding for diarrhea-predominant irritable bowel syndrome," Journal of New Chinese Medicine, vol. 51, no. 7, pp. 209-212, 2019, in Chinese.

[30] J. Wang, "60 cases of diarrhea type irritable bowel syndrome treated by Catgut Embedding at the Bamai Jiaohui Acupoints and Acupoint Application of traditional Chinese Medicine," Chinese Journal of Traditional Medical Science and Technology, vol. 24, no. 1, pp. 77-78, 2017, in Chinese.

[31] J. Yao, "Clinical study on the effect of moxibustion and catgut implantation on patients of irritable bowel syndrome of the type of diarrhea," Master's Thesis, Guangzhou University of Chinese Medicine, Guangzhou, China, 2012, in Chinese.

[32] B. Ying, G. Yu, G. Wang, and W. Huang, "26 cases of diarrhea predominant irritable bowel syndrome treated by the Herbal 
Cake moxibustion combined with Acupoint Catgut Embedding," Jiangxi Journal of Traditional Chinese Medicine, vol. 11, p. 67, 2009, in Chinese.

[33] J. Zeng, "81 cases of diarrhea type irritable bowel syndrome treated with Buzhong Yiqi Decoction and Catgut Embedding at acupoints," Guangming Journal of Chinese Medicine, vol. 28 , no. 4, pp. 763-764, 2013, in Chinese.

[34] J. Zhao, "Acupoint catgut embedding treatment of liver and spleen deficiency syndrome-diarrhea-predominant irritable bowel syndrome: clinical effect observation," Master's Thesis, Shanxi University of Chinese Medicine, Taiyuan, China, 2014, in Chinese.

[35] Z. Zhao, "Clinical study on catgut implantation at acupoint for diarrhea-predominant irritable bowel syndrome," Master's Thesis, Hebei University, Shijiazhuang, China, 2016, in Chinese.

[36] Y. Liang, Y. Zhang, and J. Feng, "Therapeutic effect of Taijiquan combined with acupoint catgut embedding on constipation type irritable bowel syndrome," Hubei Journal of Traditional Chinese Medicine, vol. 10, pp. 50-51, 2010, in Chinese.

[37] W. Liu, "Clinical and experimental study on acupoint catgut embedding therapy for constipation type irritable bowel syndrome," Doctor's Thesis, Beijing University of Chinese Medicine, Beijing, China, 2019, in Chinese.

[38] X. Gao, Y. Xu, S. Wang, and P. Sun, "Clinical observation on 33 cases of irritable bowel syndrome with constipation treated by acupoint catgut embedding and auricular point sticking," Journal of Gansu University of Chinese Medicine, vol. 33, no. 6, pp. 66-68, 2016, in Chinese.

[39] S. Chai, Y. Jin, and B. Zhou, "Clinical study on acupoint catgut embedding combined with lactulose in the treatment of constipation-type irritable bowel syndrome," Jiangsu Journal of Traditional Chinese Medicine, vol. 51, no. 8, pp. 63-65, 2019, in Chinese.

[40] H. Xu and D. Ding, "Observation on therapeutic effect of acupoint catgut embedding combined with auricular point sticking on constipation type irritable bowel syndrome," Hubei Journal of Traditional Chinese Medicine, vol. 10, pp. 35-36, 2018, in Chinese.

[41] L. Zou, "Effect of acupoint catgut embedding combined with Shugan Daozhi Decoction on constipation type irritable bowel syndrome," Master's Thesis, Liaoning University of traditional Chinese Medicine, Shenyang, China, 2010, in Chinese.

[42] H. Hao, S. Ren, Z. Liu, and N. Li, "Effect of acupoint catgut embedding combined with Zhizhu Granule on constipation type irritable bowel syndrome," Shanxi Journal of Traditional Chinese Medicine, vol. 4, pp. 30-31, 2013, in Chinese.

[43] L. Dong, "Clinical study on acupoint catgut embedding in the treatment of constipation type irritable bowel syndrome," Doctor's Thesis, Guangzhou University of Chinese Medicine, Guangzhou, China, 2011, in Chinese.

[44] G. Jin, "Efficacy of acupoints catgut embedding in treating irritable bowel syndrome of constipation type," Chinese Journal of Integrated Traditional and Western Medicine on Digestion, vol. 22, no. 1, pp. 5-7, 2014, in Chinese.

[45] Y. Liang and S. Luo, "Observations on the efficacy of finger acupuncture plus acupoint catgut embedding in treating irritable bowel syndrome of constipation type," Shanghai Journal of Acupuncture and Moxibustion, vol. 3, pp. 168-169, 2010, in Chinese.

[46] H. Hao, S. Ren, N. Li et al., "Effect of Zhizhu Decoction Combined with acupoint catgut embedding on anorectal manometry in constipation type irritable bowel syndrome," Shanxi Journal of Traditional Chinese Medicine, vol. 31, no. 12, pp. 16-18, 2015, in Chinese.

[47] J. P. T. Higgins and S. Green, "Cochrane handbook for systematic reviews of interventions version 5.1.0 [K/OL]," 2013, http://www.cochrane-handbook.org.

[48] L. Manzoli, M. E. Flacco, C. Marzuillo, and L. Lopetuso, "Prevalence of severe irritable bowel syndrome among Italian adults. A meta-analysis," European Review for Medical and Pharmacological Sciences, vol. 21, no. 24, pp. 5751-5764, 2017.

[49] J. Sheng, X. Jin, J. Zhu, Y. Chen, and X. Liu, "The effectiveness of acupoint catgut embedding therapy for abdominal obesity: a systematic review and meta-analysis," Evidence-Based Complementary and Alternative Medicine, vol. 2019, Article ID 9714313, 12 pages, 2019.

[50] L. Zhou and H. Cai, "Adjuvant treatment of acupoint catgut embedding for essential hypertension and its effects on vascular endothelial function," Zhongguo Zhen Jiu, vol. 38, no. 4, pp. 349-352, 2018.

[51] Y. Feng, Y. Fang, Y. Wang, and Y. Hao, "Acupoint therapy on diabetes mellitus and its common chronic complications: a review of its mechanisms," BioMed Research International, vol. 2018, Article ID 3128378, 9 pages, 2018.

[52] F. Huang, Y. Xie, S. Zhao, Z. Feng, G. Chen, and Y. Xu, "The effectiveness and safety of acupoint catgut embedding for the treatment of postmenopausal osteoporosis: a systematic review and meta-analysis," Evidence-Based Complementary and Alternative Medicine, vol. 2019, Article ID 2673763, 13 pages, 2019.

[53] W. Qin, K. Zhao, and H. Yang, "Effect of acupoint catgut embedding therapy combined with Chinese medicine for nourishing the kidneys and promoting blood circulation and improving blood glucose and lipid levels as well as the pregnancy rate in obese PCOS patients with infertility," $E x$ perimental and Therapeutic Medicine, vol. 12, no. 5, p. 2909, 2016.

[54] S. Yang, J. Wu, Q. Zhang et al., "Catgut implantation at acupoint reduces immune reaction in a rat model of allergic rhinitis," Evidence-Based Complementary and Alternative Medicine, vol. 2018, Article ID 7629239, 10 pages, 2018.

[55] B. B. Ren, Z. Yu, and B. Xu, "Overview of the two-way regulatory effect of acupuncture on gastrointestinal motility," Zhongguo Zhen Jiu, vol. 32, no. 8, pp. 765-768, 2012.

[56] J. F. Jiang, L. L. Wang, X. Bin, H. Ling, X. G. Song, and H. G. Wu, "Anti-inflammatory: effect mechanism of warming-dredging in moxibustion," Zhongguo Zhen Jiu, vol. 33, no. 9, pp. 860-864, 2013.

[57] X. Xiong, W. Peng, L. Chen et al., "Traditional Chinese medicine Zhiqiao-Houpu herb-pair induce bidirectional effects on gastric motility in rats," Journal of Ethnopharmacology, vol. 175, pp. 444-450, 2015.

[58] Q. Guo, J. Li, and H. Lin, "Effect and molecular mechanisms of traditional Chinese medicine on regulating tumor immunosuppressive microenvironment," BioMed Research International, vol. 2015, Article ID 261620, 12 pages, 2015.

[59] J. Huo, J. Zhao, Y. Yuan, and J. Wang, "Research status of the effect mechanism on catgut-point embedding therapy," Zhongguo Zhen Jiu, vol. 37, no. 11, pp. 1251-1254, 2017.

[60] X. P. Zhang, C. S. Jia, J. L. Wang et al., "Acupoint catgutembedding therapy: superiorities and principles of application," Zhongguo Zhen Jiu, vol. 32, no. 10, pp. 947-951, 2012.

[61] S. Zhang, Q. Li, W. Wei, and Y. Lai, "Consensus on standard management of irritable bowel syndrome in TCM," China 
Journal of Traditional Chinese Medicine and Pharmacy, vol. 25, no. 7, pp. 1062-1065, 2010.

[62] C. Huang and W. Pan, "Comparation of effect and costbenefit analysis between acupoint catgut-embedding and electroacupuncture on simple obesity," Zhongguo Zhen Jiu, vol. 31, no. 10, pp. 883-886, 2011.

[63] J.-W. Yang, L.-Q. Wang, X. Zou et al., "Effect of acupuncture for postprandial distress syndrome," Annals of Internal Medicine, vol. 172, no. 12, p. 777, 2020.

[64] Y. He, X. Guo, B. H. May et al., "Clinical evidence for association of acupuncture and acupressure with improved cancer pain," JAMA Oncology, vol. 6, no. 2, p. 271, 2020.

[65] L. Zhao, D. Li, H. Zheng et al., "Acupuncture as adjunctive therapy for chronic stable Angina," JAMA Internal Medicine, vol. 179, no. 10, p. 1388, 2019.

[66] Q. Fu, H. Yang, L. Zhang et al., "Traditional Chinese medicine foot bath combined with acupoint massage for the treatment of diabetic peripheral neuropathy: a systematic review and meta-analysis of 31 RCTs," Diabetes/Metabolism Research and Reviews, vol. 36, no. 2, p. e3218, 2020.

[67] X. L. Wang, G. H. Lin, N. Xu, J. C. Zeng, D. H. Xu, and S. X. Wang, "Analysis of adverse reactions of acupoint catgut embedding therapy," Zhongguo Zhen Jiu, vol. 40, no. 2, pp. 193-196, 2020.

[68] Q. Wang, Clinical Analysis on Acupoint Catgut-Embedding Therapy for Abdominal Obesity, Guangzhou University of Chinese Medicine, Guangzhou, China, 2008. 